\title{
OPTIMIZATION OF DIFFUSER VALVES
}

\section{Chin-Tsan Wang}

Department of Mechanical and Electro-Mechanical Engineering, National, I-Lan University, I-Lan, Taiwan, R.O.C., ctwang@niu.edu.tw

\section{Pi-Cheng Chang}

Department of Mechanical and Electro-Mechanical Engineering, National, I-Lan University, I-Lan, Taiwan, R.O.C.

Chien-Cheng Huang

Department of Mechanical and Electro-Mechanical Engineering, National, I-Lan University, I-Lan, Taiwan, R.O.C.

Follow this and additional works at: https://jmstt.ntou.edu.tw/journal

Part of the Mechanical Engineering Commons

\section{Recommended Citation}

Wang, Chin-Tsan; Chang, Pi-Cheng; and Huang, Chien-Cheng (2008) "OPTIMIZATION OF DIFFUSER VALVES," Journal of Marine Science and Technology. Vol. 16: Iss. 2, Article 5.

DOI: $10.51400 / 2709-6998.2023$

Available at: https://jmstt.ntou.edu.tw/journal/vol16/iss2/5

This Research Article is brought to you for free and open access by Journal of Marine Science and Technology. It has been accepted for inclusion in Journal of Marine Science and Technology by an authorized editor of Journal of Marine Science and Technology. 


\title{
OPTIMIZATION OF DIFFUSER VALVES
}

\author{
Chin-Tsan Wang*, Pi-Cheng Chang*, and Chien-Cheng Huang*
}

Key words: diffuser valve, optimal strouhal number.

\begin{abstract}
In this study, the width of channel, $\mathrm{D}$, and diffuser angle, $\alpha$, at given diffuser width whose value is $100 \mu \mathrm{m}$ will be studied for steady and unsteady flow conditions. By means of a series of numerical simulations, some findings for diffuser valve will be reported. The diffuser valve with a channel width of $\mathrm{D}=300 \mu$ $\mathrm{m}$ and diffuser angle $\alpha=60^{\circ}$, is a better geometry because of its outstanding capability on suction production of net volume flow. Besides, the larger the Reynolds number is, the greater the suction amount of net volume flow will be. When the aspect ratio (AR) is approaching to the value of 4 , the diffuser valve becomes more effective. An optimal Strouhal number, $S t_{H}$, based on production of maximum net volumetric flow is 0.338 for $\mathrm{D}=300 \mu \mathrm{m}$. These findings will be useful to micropump of diffuser valve.
\end{abstract}

\section{INTRODUCTION}

Research on micropumps was initiated in 1980 and numerous different pumps have since been developed [5]. It would be useful at this point to actually quote the major players here.

Numerous fluidic applications in such areas as medicine, chemistry, environmental testing and thermal transport have the potential to be "scaled down" for reasons of sample size, device cost or portability. Cost-effective, yet capable and reliable fluidic components (including pumps) are required for such scaled down systems. Currently, many pump designs are based on valves that "open and close". While such valves are often found in macroscopic applications, the discrete nature of their operation is not necessarily the best choice for micro-applications. These pumps require valve sets or other type of sealing and anti-seizure mechanisms and typically are limited to fully-opened clearances that are significantly smaller than fixed-geometry valves.

Micropumps that have been developed are based on various principles of actuation and contain various types of valves. For a typical micropump, an actuation component with two microvalves is required. Piston or membrane structure for actua-

Paper submitted 01/09/07; accepted 03/05/07. Author for correspondence: Chin-Tsan Wang (e-mail: ctwang@niu.edu.tw).

*Department of Mechanical and Electro-Mechanical Engineering, National, I-Lan University, I-Lan, Taiwan, R.O.C. tion is commonly used to generate the necessary driving force in the pump. The microvalves structure can appear in several forms or categories and these are defined as active or passive microvalves $[3,4,7]$. Active microvalves are actuated by using external forces to open or close the valve seats. One example is the electrostatic type reported by Ohnstein T [8].

Passive microvalves are designed with or without moving parts. For the passive microvalves with moving parts, the valve acts like a one-way valve that opens in one flow direction and closes in the other (essentially a check valve). In contrast, passive microvalves with no moving parts do not actually open and close the valve seats. NO-moving parts valve (NMPV) produce a net flow resulted from pressure resistance in forward and reverse flow. Both active and passive microvalves with moving parts are expensive and complicated in their production and maintenance. Further, it has been shown that active/passive valves with moving parts can easily cause damage to biological particles in the fluid if the pump is used in the transport of biological samples. However, NMPV micropumps are not subjected to such constraints because of their simplicity, reliability, ease of manufacture, and potential for being able to pass biological particles. As a result, these pumps have found numerous applications in medicine, chemistry, and environmental testing.

The first valve-less diffuser pump was introduced in 1993 [12, 13]. Since then many types of NMPV's have been developed. The Tesla valve is one example that has found many applications in industry because of its simplicity of geometry [14]. Its operation is based on the principle of pressure drop in the inlet for forward and reverse flow. A second type is a diffuser valve which is a simple flat diffuser oriented such that the forward flow sees diverging walls and the reverse flow sees converging walls. Forster [10] presents techniques for design and testing of NMPV including the Tesla-type and the diffuser valve. Gerlach [2] and Olsson [15] focus their research solely on diffuser valves.

A micropump with two diffuser valves embedded in the inlet and outlet positions of the channel was reported [4, 12]. The flow has lower flow resistance along the diffuser direction and larger flow resistance along the convergent nozzle flow direction for a diffuser valve with angle less than $20^{\circ}$. However, these conclusions were based on studies undertaken at steady state flow conditions. Besides, an investigation of flat-walled diffuser elements for valve-less micropumps is presented by Olsson et al [9]. The diffuser element is a small angle flow channel with a rounded inlet and a preferably sharp outlet. The diverging-wall direction is the positive flow direction. The flow-directing capability under steady flow conditions was determined experimentally for several different diffuser elements. The simu- 
lations show the flow-directing capability of the diffuser elements and predict the flow-pressure characteristics well for Reynolds numbers below 300-400. The suitability of valve-less micropumps in biochemistry has been addressed by Andersson et al. [1]. The results show that the valve-less micropump successfully pumps fluids within the viscosity range of $0.001-0.9 \mathrm{~N} \mathrm{~s} / \mathrm{m}^{2}$. The micropump is not sensitive to the density, ionic strength, or $\mathrm{pH}$ of the pumped media. Finally, a new concept of designing microfluidic devices using printed circuit board (PCB) as the substrate material have been successfully developed for miniature valveless pumps. The pump can be operated as a single diffuser/nozzle pump or a peristaltic pump, and can delivery a maximum flow rate of $3 \mathrm{ml} / \mathrm{min}$ [6].

However, few studies of unsteady effects on the microvalves with no-moving parts have been done. There had been very few literatures discussing its optimization. The valve's geometry in the microchannel has not been reported. This lack of information has hindered the application of NMPV. In this paper, the performance of NMPVs for diffuser valve under both steady and unsteady flow conditions will be presented using numerical simulation approaches. Optimal operation parameters for diffuser valve of NMPV micropump design are addressed in the study.

\section{NUMERICAL METHOD AND GEOMETERIC MODEL}

The simulation was performed with the CFD-ACE+ software (CFD Research Corporation, Huntsville Alabama), a multi-physics package which is based on the Finite-Volume method. The program was run on a $2.4 \mathrm{Ghz}$ Pentium IV processor with 1 GB of RAM memory. Mesh-independent tests were established in initial feasibility studies; a mesh range of $\mathrm{N}=1552$ to 13328 cells were adopted for the 2D flow simulation, but enhancing the accuracy $\mathrm{N}$ was chosen to be 15561 . For the $3 \mathrm{D}$ flow simulation to establish the aspect ratio effect, the total cell numbers was $\mathrm{N}=497952$. The structured grid method was used for dealing with the complexity of 3D flow simulations. Although the valve flow was laminar, a rather fine mesh was needed to account for the detailed features of the sorting mechanism. The time for each run spanned from 2 hours up to 12 hours.

Details of the model implemented in CFD-ACE+ software is now addressed. The governing equations for the flow model represent mathematical statements of the mass and momentum conservation laws. These two laws were used to develop a set of equations (known as the Navier-Stokes equations) for $\mathrm{CFD}-\mathrm{ACE}+$ to solve numerically.

\section{Mass Conservation}

Conservation of mass requires that the time rate of change of mass in a control volume must be balanced by the net mass flow into the same control volume (outflow - inflow). This can be expressed as:

$$
\frac{\partial \rho}{\partial t}+\nabla \bullet(\rho \vec{V})=0
$$

a)

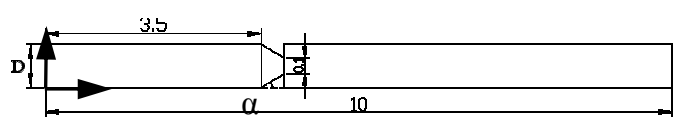

b)

Fig. 1. Diagram of microchannel for no-moving-parts valve (NMPV). D is width of channel, $\alpha$ is diffuser angle and $H$ is width of diffuser whose value is $100 \mu \mathrm{m}$.

where $\rho$ is the density of fluid and $\vec{V}$ is velocity vector of the flow field.

\section{Momentum Conservation}

Newton's second law states that the time rate of change of the momentum of a fluid momentum equation is found by setting the rate of change of $\mathrm{x}, \mathrm{y}$, and $\mathrm{z}$-momentum of the fluid particle equal to the total force in the $\mathrm{x}, \mathrm{y}$, and $\mathrm{z}$-direction on the element due to surface stresses plus the rate of increase of $x, y$, and $\mathrm{z}$-momentum due to sources. The Navier-Stokes equations can be simply expressed as:

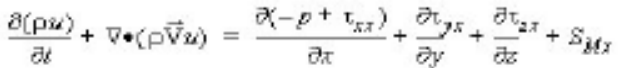

$$
\begin{aligned}
& \frac{\partial(p v)}{\partial t}+\nabla \cdot(p \vec{v} v)=\frac{\partial \tau_{y y}}{\partial x}+\frac{\partial(-p+\zeta y}{\partial y}+\frac{\partial \tau_{x y}}{\partial z}+s_{j g} \\
& \frac{\partial(\rho w)}{\partial t}+\nabla \bullet(\rho \vec{V} w)=\frac{\partial \tau_{x z}}{\partial x}+\frac{\partial \tau_{y z}}{\partial y}+\frac{\partial\left(-p+\tau_{z z}\right)}{\partial z}+S_{M z}
\end{aligned}
$$

where $\vec{V}=u \vec{i}+v \vec{j}+w \vec{k}, \mathrm{u}, \mathrm{v}, \mathrm{w}$ are velocity vectors in $\mathrm{x}, \mathrm{y}$, and $\mathrm{z}$ direction. $\tau_{i j}$ is stress tensor, $\mathrm{p}$ is pressure and $\mathrm{S}_{\mathrm{M}}$ is the external source term.

A duct channel with no-moving valve for diffuser valve, shown in Fig. 1, will be used because of its simple prototype and appropriately apply the above equations to an element in microchannel.

In developing the mesh, a triangular structured Grid avoided the problem of singular geometrical points. The total numbers of cell were 15561 and 497952 for the 2D and 3D flow simulations, respectively. The convergent condition was assumed to be $\pm 10^{-20}$ for the numerical simulations. On the boundary condition of simulation process, a constant inlet velocity calculated from a given Reynolds number whose values are from 0.01 to 100 will be used for steady analysis. But the inlet driving pressure $\mathrm{P}$ defined as $\mathrm{P}=\mathrm{P}_{0} \sin (2 \pi \mathrm{f} * \mathrm{t})$ will be applied to unsteady analysis. Here, the magnitude of inlet driving pressure $\mathrm{P}_{0}$ will be set as 400,500 and $600 \mathrm{~Pa}$, respectively. The $\mathrm{f}$ is the driving frequency and $t$ is the time.

The numerical model of NMPV for a given diffuser width $\mathrm{H}=100 \mu \mathrm{m}$ and total channel length $\mathrm{L}=10 \mathrm{~mm}$ (see Fig. 1) was initially considered. Varying geometric parameters such as the diffuser angle and channel width facilitated an optimization to the design of the diffuser valve for NMPV structure. The diffuser angle, $\alpha$, was set as $20^{\circ} 、 40^{\circ}$ and $60^{\circ}$ and the channel width, D, was chosen as $200 \mu \mathrm{m}$ and $300 \mu \mathrm{m}$ for $2 \mathrm{D}$ simulation. The 
flow direction along the positive $\mathrm{X}$ axis was defined as the forward flow and vice versa for negative flow.

To realize the effect of net volume flow, an index defined as $D_{i}^{p}$ was used and mathematically stated as:

$$
D_{i}^{p}=\frac{\Delta p_{r}}{\Delta p_{f}}
$$

where, $\triangle \mathrm{P}_{\mathrm{f}}$ is the pressure drop of the forward flow direction and the $\triangle P_{r}$ is the pressure drop of the reverse flow in the inlet under the same flow rate condition.

When the value of $D_{i}^{p}$ of NMPV equal one, the net volumetric flow will be zero. When $D_{i}^{p}$ is greater than one and increases, the volumetric flow rate increases.

According to [12], a new parameter $\chi$ called rectification efficiency can be used and defined as:

$$
\chi=\left(\sqrt{D_{i}^{p}}-1\right) /\left(\sqrt{D_{i}^{p}}+1\right)
$$

Here, the positive value of $\chi$ corresponding to $D_{i}^{p}>1$ indicates a pumping performance. Oppositely, the negative value of $\chi$ corresponding to $D_{i}^{p}<1$ indicates a suction performance of diffuser valve.

This index $\chi$ will better describe the rectification effect and be used to study the performance of diffuser valve in this study

\section{NUMERICAL RESULTS AND DISCUSSIONS}

The main purpose of the study is to find optimal design and operational conditions for the diffuser valve of NMPV. Hence, the effect of Reynolds number on the value of $\chi$ is investigated over the range of $\operatorname{Re}=0.01$ to 80 for steady flow conditions. Typical results for this study are shown in Fig. 2. The pressure drops were evaluated from the CFD simulation by averaging the numerical pressures at the outlet of channel. The values of $\chi$ are all made less than zero. That is to say, this condition means the diffuser valve acts as a suction ability of a pump. General speaking, larger Reynolds number resulted in smaller values of $\chi$. It implies to possess greater suction capabilities of the pump. For lowest value of $\chi$ (specifically, the case of $\mathrm{D}=300$ $\mu \mathrm{m}$ and $\alpha=60^{\circ}$ ) the net suction volumetric flow was maximized.

A typical flow image for the diffuser valve is shown in Fig. 3. It can be observed that flow separation occurs at $\mathrm{Re}=80$ and the flow field becomes asymmetric. This asymmetric flow phenomenon becomes more prominent with an increase in channel width $\mathrm{D}$ and diffuser angle $\alpha$. This asymmetry can also induce a different pressure drop for the forward and reverse flow passing through the diffuser valve at different Re. Therefore, the value of $\chi$ becomes much less than zero as Re increases and the suction performance becomes more effective.

In the more "realistic" microfluid condition, the viscous force and surface tension force will influence the operational efficiency of the micropump. To account for this, a special family parameter defined as the aspect ratio, AR (W/H), shown in Fig.

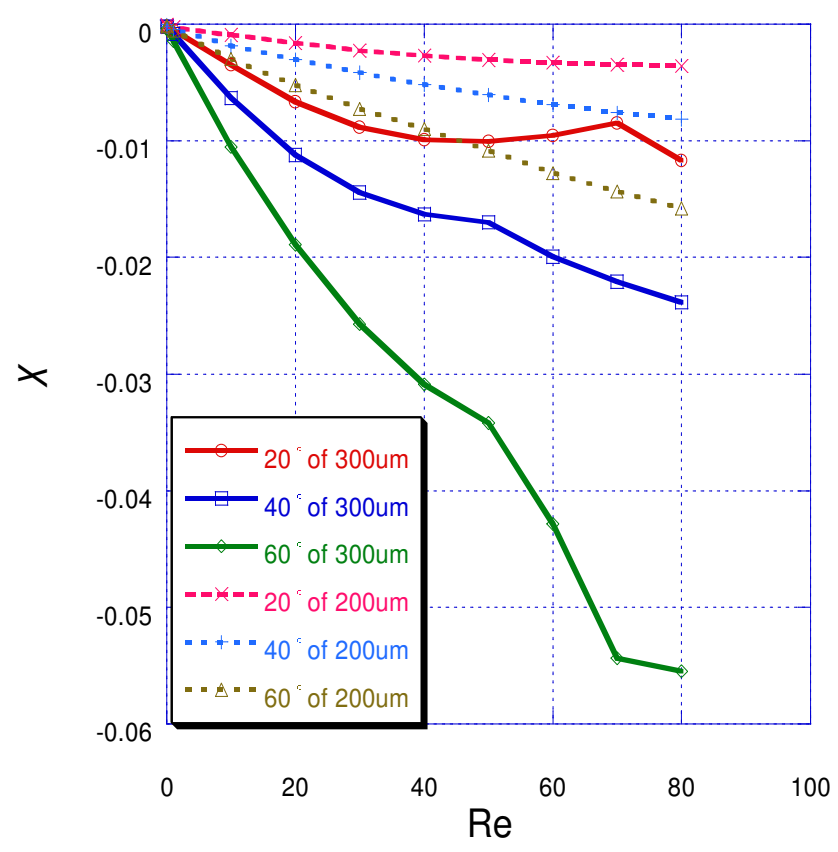

Fig. 2. The $\chi$ versus Reynolds number for different diffuser angle $\alpha$ at $\mathrm{D}=200 \mu \mathrm{m}$ and $300 \mu \mathrm{m}$, respectively.

(a) $\operatorname{Re}=0.01$

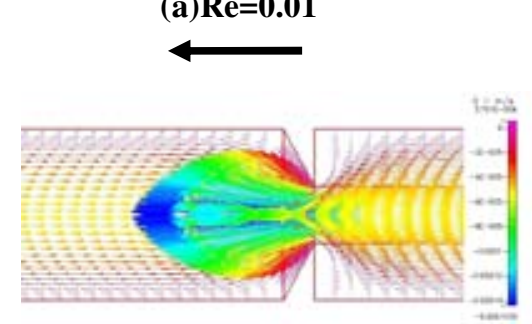

(b) $\operatorname{Re}=80$

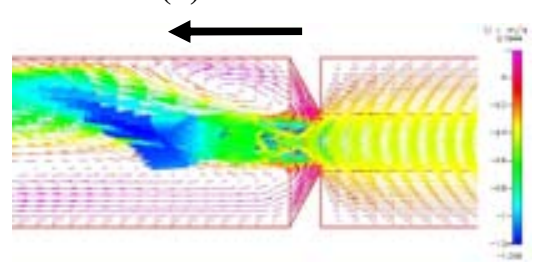

Fig. 3. The flow image was taken at (a) $\mathrm{Re}=0.01$ and (b) $\mathrm{Re}=80$ for the diffuser angle $\alpha=60^{\circ}$ and width of channel $D=300 \mu \mathrm{m}$.

1 is used. Here $\mathrm{W}$ is the depth of channel in the $\mathrm{Z}$ direction and $\mathrm{H}$ is the width of diffuser whose value is $100 \mu \mathrm{m}$.

In Fig. 4 , it is apparent that the value of $\chi$ at $\mathrm{AR}=4$ will approach the results of a $2 \mathrm{D}$ flow simulation because the effect of viscous force is unobvious and could be neglected. An example of three dimensional flow image will be taken for explaining the effect of Aspect ratio at $\mathrm{Re}=60$ and $\mathrm{AR}=2$ for the case of channel width $\mathrm{D}=300 \mu \mathrm{m}$ and diffuser angle $\alpha=60^{\circ}$. On the flow image of Fig. 5, the three dimensional flow structures originated from the side-wall effect are seen clearly.

To imitate the realistic operation of piezoelectric buzzer driving force system, a sine wave with the different amplitude 


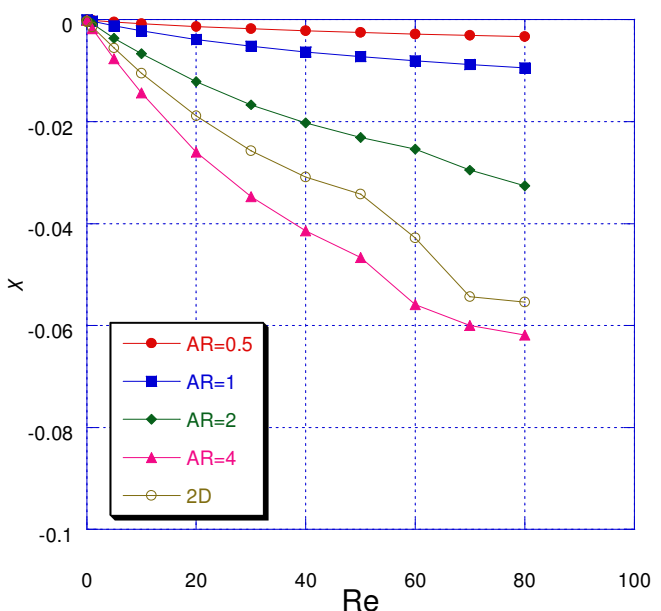

Fig. 4. The $\chi$ versus Reynolds number at different aspect ratio (AR) for the 2D simulation as well as the case of channel width $\mathrm{D}=300 \mu \mathrm{m}$ and diffuser angle $\alpha=60^{\circ}$.

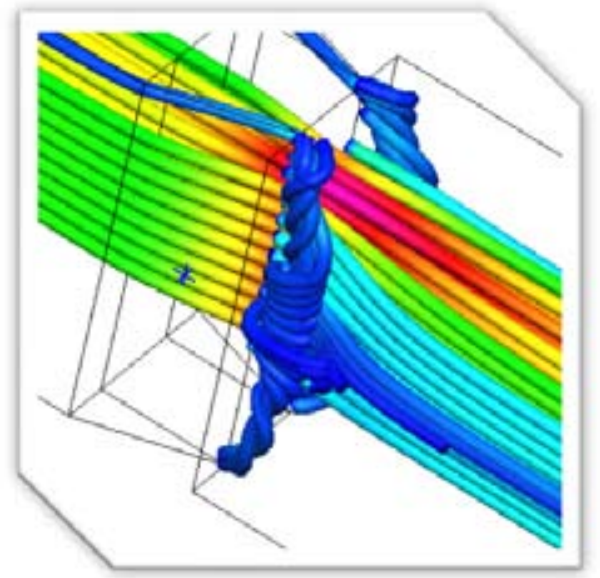

Fig. 5. A 3D image of flow was taken at $R e=60$ and $A R=2$ for the case of channel width $D=300 \mu \mathrm{m}$ and diffuser angle $\alpha=60^{\circ}$.

and frequency corresponding to the different pressure energy and driving frequency are applied at the inlet of microchannel to study the unsteady effects on the net volume flow and the optimal operation for different flow conditions. Here, the driving frequency and the net volume flow are the two most important parameters on studying the efficiency of diffuser valve for NMPV micropumps. Hence, a nondimensional parameter for driving frequency $\mathrm{f}$, names as Strouhal number $S t_{H}$, is defined as

$$
S t_{H}=\frac{f \cdot H}{U_{\text {ave }}}
$$

where $f$ is the driving frequency, $H$ is the throat width of the diffuser and $U_{\text {ave }}$ is the average velocity for the net volumetric flow at the throat area of the diffuser valve.

The Strouhal number is a parameter which facilitates the determination of the maximum net volume flow in a practical operation. This maximum flow condition is defined as $S t_{H}$. The normalized mass flow rate and the normalized mass flow rate per period as a function of the Strouhal number for various inlet pressures are shown in Fig. 6. Generally speaking, the greater

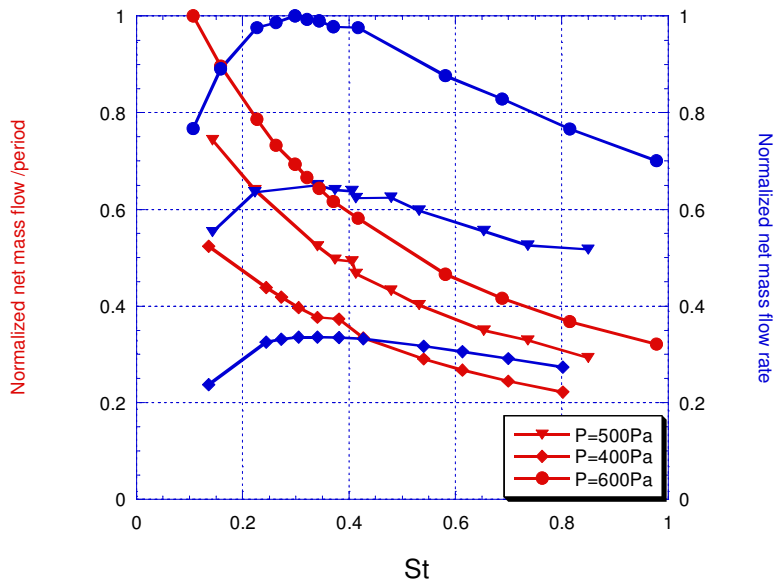

Fig. 6. The Strouhal number versus the net nondimsionalized volumetric flow rate and nondimsionalized volumetric flow rate per period for inlet pressures at the case of channel width $\mathrm{D}=300 \mu \mathrm{m}$ and diffuser angle $\alpha=60^{\circ}$.

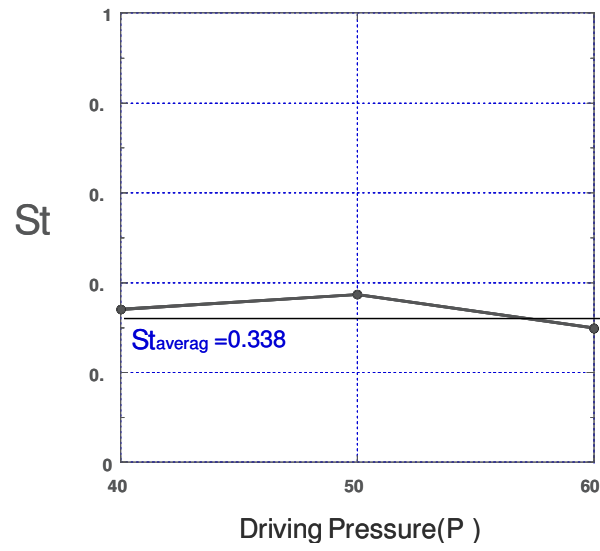

Fig. 7. The Strough number versus inlet pressure amplitude for different diffuser throat widths.

the pressure energy at the inlet, the greater the net volumetric flow is. It is observed that the mass flow rate per period decreases with an increase in value of St because of fluid inertia. It is further observed that the net flow rate is related to not only the flow per period, but also to the driving frequency. Hence, a maximum in the net volumetric flow rate occurs and results in a value of St which is fairly independent of the inlet pressure. An optimal value of $S t_{H}=0.338$ was determined as shown in Fig. 7 . Given the diffuser width, $\mathrm{H}$, the maximum volume flow rate and optimal driving frequency can readily be estimated using the optimal value of $S t_{H}=0.338$. This procedure is very important to the design of diffuser valve for NMVP's pumps.

\section{CONCLUSION}

Using a numerical CFD approach, some important results for the optimal design of diffuser valve has been presented. First, the value of $\chi$ is less than zero with Re studied. That is to say, it would produce net volume flow by way of suction. This study has ascertained that a channel width $\mathrm{D}=300 \mu \mathrm{m}$ and diffuser 
angle $\alpha=60^{\circ}$ is a good choice for design because it results in a maximum condition for output net volumetric flow. The optimal Strouhal number $S t_{H}=0.338$ was determined and found to be an effective nondimensional parameter which could be useful for optimal design of diffuser valve.

\section{REFERENCES}

1. Andersson, H., Van Der Wijngaart, W., Enoksson, P. and Stemme, G., "Valve-less diffuser micropump for microfluidic analytical systems," Sensors and Actuators, B: Chemical, Vol. 72, No. 3 , pp. 259-265 (2001).

2. Büstgens,B., Bacher, W., Menz, W., and Schomburg, W. K., "Micropump manufactured by thermoplastic molding," IEEE 7th International Workshop on Micro Electro Mechanical Systems (MEMS'94), Oiso, Japan, pp. 18-21 (1994).

3. Fuhr, G., Hagedorn, R., Muller, T., Benecke, W., and Wagner, B., "Pumping of water solution in microfabricated electrohydrodynamic System," Proc. IEEE-MEMS Workshop, pp. 25-29 (1992).

4. Gerlach, T., Schuenmann, M., and Wurmus, H., "A new micropump principle of the reciprocation type using pyramidic micro flow channels as passive valves," Journal of Micromechanics and Microengineering, Vol. 5, pp. 199-201 (1995).

5. Gravesen, P., Brandebjerg, J., and Søndergard Jensen, O., "Microfluidics - a review," Journal of Micromechanics and Microengineering, Vol. 3 , pp.168-182 (1993).

6. Nguyen, N. T., Huang, X., "Miniature valveless pumps based on printed circuit board technique," Sensors and Actuators, A: Physical, Vol. 88, No. 2 , pp. 104-111 (2001)

7. Nguyen, N. T., Huang, X., Chuan, T. K., "MEMS-micropumps: a review," Transactions of the ASME Journal of Fluids Engineering, Vol. 124, No. 2 , pp. 384-392 (2002).

8. Ohnstein, T., Fukjura, T., Ridley, J., and Bonne, U., "Micromachined silicon microvalve," Proc. IEEE-MEMS Workshop, pp. 95-98 (1990).

9. Olsson, A., Stemme, G. and Stemme, E., "Numerical and experimental studies of flat-walled diffuser elements for valve-less micropumps," Sensors and Actuators, A: Physical, Vol. 84, No. 1, pp. 165-175 (2000).

10. Schomburg, W. K., Vollmer, J., Büstgens, B., Fahrenberg, J., Hein, H., and Menz, W., "Microfluidic components in LIGA technique," Journal of Micromechanics and Microengineering, Vol. 4, pp. 186-191 (1994).

11. Shoji, S., and Esashi, M., "Microflow devices and system," Journal of Micromechanics and Microengineering, Vol. 4, pp.157-171 (1994).

12. Stemme, E. and Stemme G., "A valve-less diffuser/nozzle based fluid pump," Sensors and Actuators, Vol. A39, pp. 159-167 (1993).

13. Stemme, E. and Stemme, G., "Valve-less fluid pump," Swedish Patent Appl., No. 9 300, pp.604-7, (1993).

14. Tesla, N., "Valvular conduit," United States Patent US1 (1920).

15. Zengerle, R., Kluge, S., Richter, M., and Richter, A., "A bidirectional silicon micropump," IEEE 8th International Workshop on Micro Electro Mechanical Systems (MEMS'95), Amsterdam, the Netherlands, pp. 19-24 (1995). 